\title{
Tratamiento médico de la enuresis en niños: aspectos actuales
}

Salazar-Ozuna WA, Aragón-Castro MA, Vázquez-Niño CL, Ruvalcaba-Oceguera G, Gutiérrez-Rosales R, Téllez-Díaz Trujillo JA

\section{Resumen}

La enuresis es un trastorno frecuente en todo el mundo; sin embargo, es anormal después de los 5 años de edad, que resulta en aislamiento social y alteraciones en el núcleo familiar. El objetivo de este estudio es reportar los aspectos actuales del tratamiento de la enuresis. La fisiopatología de este trastorno varía desde alteraciones en la capacidad vesical, cantidad de orina producida durante la noche e incapacidad del niño para levantarse y acudir al sanitario. Parte del tratamiento consiste en establecer el diagnóstico adecuado y motivar al niño y sus padres a implementar el tratamiento ideal, mediante terapias conductuales (como la técnica de alarma) o farmacológicas con desmopresina e imipramina. El seguimiento se realiza por 3 meses, con la suspensión del mismo durante una semana, para valorar y determinar si debe omitirse permanentemente o continuar con la dosis mínima efectiva hasta lograr episodios secos por las noches.

PALABRAS CLAVE: enuresis, desmopresina, alarma, imipramina.

Rev Mex Urol. 2017 may;77(3):230-237.

\section{Medical management of enuresis in children: current aspects}

Salazar-Ozuna WA, Aragón-Castro MA, Vázquez-Niño CL, Ruvalcaba-Oceguera G, Gutiérrez-Rosales R, Téllez-Díaz Trujillo JA

\begin{abstract}
Enuresis is a frequent pathology in all cultures, but is abnormal after 5 years of age, contributing to conditions of social isolation in the child and alterations in the family nucleus. The aim of this study was to report on current aspects of enuresis treatment. The pathophysiology of this disorder ranges from bladder capacity alterations, the quantity of urine produced during the night, and the incapacity of the child to wake up and use the bathroom. Part of the treatment is the correct diagnosis and precise motivation of both the child and parents to carry out the ideal management, utilizing behavioral therapies, such as setting a wake-up alarm, or drug therapies, such as desmopressin and imipramine. There is a 3-month follow-up with treatment suspension for one week to evaluate and determine whether treatment can be permanently suspended or continued with a minimum effective dose, achieving the maintenance of dry nocturnal episodes.
\end{abstract}

KEY WORDS: Enuresis; Desmopressin; Alarm; Imipramine
Departamento de Urología, Instituto de Seguridad Social del Estado de México y Municipios, Toluca, Estado de México, México.

Recibido: octubre 2016

Aceptado: febrero 2017

Correspondencia

Dr. Wilberth Antonio Salazar Ozuna

waso_186@hotmail.com

drwsalazarozuna@gmail.com

Este artículo debe citarse como

Salazar-Ozuna WA, Aragón-Castro MA, VázquezNiño CL, Ruvalcaba-Oceguera G, Gutiérrez-Rosales R, Téllez-Díaz Trujillo JA. Tratamiento médico de la enuresis en niños: aspectos actuales. Rev Mex Urol. 2017 mayo;77(3):230-237. 


\section{ANTECEDENTES}

\section{Enuresis}

De acuerdo con la Sociedad Internacional de Continencia en Niños, el concepto actual de enuresis se refiere a la incontinencia urinaria intermitente (fuga de orina en discreta cantidad), exclusivamente durante periodos de sueño, significativo en la frecuencia con el ICD-10 (micción involuntaria por las noches, mínimo durante 3 meses, con edad mental donde la enuresis es inaceptable; al menos 2 veces al mes en pacientes menores de 7 años y al menos una vez al mes en los mayores de 7 años de edad sin alteraciones neurológicas) y DSM-V (micción solamente en la noche), en el cual la frecuencia corresponde a más de 4 episodios a la semana y la infrecuencia corresponde a menos de 4 episodios a la semana. ${ }^{1}$

Este trastorno se divide en monosintomático y no monosintomático. El primero se define como la enuresis en niños sin ningún síntoma adicional del tracto urinario inferior, ni antecedente de disfunción vesical; mientras que el segundo es aquel que genera síntomas del tracto urinario inferior o con antecedente de alteraciones vesicales diurnas. ${ }^{2}$

\section{Prevalencia}

La enuresis es un problema común en niños y adolescentes (Cuadro 1). ${ }^{3-5} \mathrm{El}$ único estudio analítico en niños monosintomáticos es el ensayo británico ALSPAC, efectuado en 8,242 niños de $7 \frac{1 / 2}{2}$ años de edad. Ese estudio reportó que $15.5 \%$ de todos los pacientes mojaba la cama y de ellos, $2.6 \%$ lo hacía 2 o más noches por semana. ${ }^{6}$ Se ha observado que un tercio de los pacientes que en la infancia tuvieron enuresis sufrirán nicturia, un cuarto tendrán algún tipo de incontinencia y un quinto poseerán datos de urgencia en la edad adulta. ${ }^{7}$
Cuadro 1. Prevalencia

\begin{tabular}{|c|c|}
\hline Edad (años) & Prevalencia (\%) \\
\hline 5 & 16 \\
6 & 13 \\
7 & 10 \\
\hline 8 & 7 \\
\hline 10 & 5 \\
\hline $12-14$ & $2-3$ \\
\hline
\end{tabular}

\section{Fisiopatología}

La causa de enuresis nocturna puede resultar de uno o varios factores combinados en el niño. A continuación se analizan los de mayor trascendencia.

\section{Retraso en la maduración}

En la mayoría de los casos la enuresis nocturna desaparece de forma espontánea, por lo que el retraso en la maduración puede influir en su evolución. Algunos estudios han demostrado aumento en la incidencia de retraso en el lenguaje y desarrollo motor en niños con enuresis. ${ }^{8}$

Los episodios de enuresis se relacionan con características en el electroencefalograma (EEG) y estudios urodinámicos como: reconocimiento del llenado vesical y maduración progresiva de la estabilidad vesical, respectivamente; sin embargo, los pacientes no tienen la capacidad de suprimir la contracción vesical. ${ }^{9}$ Estos hallazgos demuestran que el retraso en la maduración interviene en el control vesical para la micción por las noches.

\section{Disfunción vesical}

Al nacimiento la capacidad vesical es de $60 \mathrm{~mL}$, aproximadamente, y con la edad incrementa $30 \mathrm{~mL}$ por año durante la niñez. En relación con esto, 
se ha observado que los niños con enuresis poseen una capacidad vesical menor que los niños sanos. ${ }^{10}$ Otro estudio reportó que la capacidad vesical máxima durante el día fue similar en los niños con enuresis y en los controles; sin embargo, en quienes padecen enuresis el volumen máximo de micción durante la noche fue significativamente menor que la capacidad vesical máxima del día, lo que sugiere la incapacidad para retener orina durante los periodos de sueño. ${ }^{11}$

En el plano urodinámico, no existe un patrón de anormalidades en niños monosintomáticos con enuresis versus niños con incontinencia diurna, quienes demuestran varias alteraciones funcionales del músculo detrusor. ${ }^{12}$

\section{Poliuria nocturna: función de la vasopresina (ADH)}

La sobreproducción de orina durante la noche, debido al desequilibro homeostásico, es bien establecida como causa de enuresis, pues existe liberación circadiana inadecuada de vasopresina en la hipófisis posterior al iniciar el ciclo del sueño y disminución en su secreción o respuesta. ${ }^{13}$ Actualmente se ha observado que en los pacientes con enuresis disminuye el ritmo circadiano de la uresis nocturna, excreción de sodio y filtración glomerular; sin embargo, este hallazgo carece de evidencia para soportar que la $\mathrm{ADH}$ es el único factor implicado. ${ }^{14}$ Hasta el momento se desconocen las razones del inadecuado ciclo circadiano y los factores que lo producen, el aumento en la reacción de tratamiento y por qué el niño es incapaz de despertarse o levantarse para la micción al manifestar estas alteraciones.

\section{Alteraciones del sueño}

Aún se discute la relación entre enuresis y anormalidades del sueño. Los padres del paciente con enuresis lo describen como un sueño excesivamente profundo. Un estudio efectuado en un laboratorio del sueño, con 33 niños de 7 a 12 años de edad ( 15 con enuresis y 18 controles), mostró que los intentos de despertarse fueron más exitosos en el grupo control. ${ }^{15}$ Además, el sueño profundo puede influir en la enuresis de adolescentes adultos. ${ }^{16}$ También se han identificado niños con enuresis durante las fases tempranas del sueño, mientras que otros estudios muestran patrones del sueño similares en niños con y $\sin$ enuresis. ${ }^{17}$

\section{Factores adicionales}

El antecedente familiar de enuresis representa otro factor asociado, lo que sugiere una causa genética. ${ }^{18}$ Estudios adicionales demuestran que el estrés y los problemas de conducta y emocionales se relacionan con enuresis. ${ }^{19}$

\section{Evaluación}

La relación entre el médico, el paciente y sus padres es parte fundamental de la evaluación clínica. Deben evaluarse diferentes aspectos (Cuadro 2); ${ }^{3,10,20,21}$ parte del protocolo incluye: examen general de orina (EGO) para valorar cetoacidosis diabética, diabetes insípida, intoxicación por agua o infección del tracto urinario. ${ }^{10}$ El urocultivo se realiza solo en caso de encontrar leucocitos o nitritos en el EGO.

El ultrasonido renal y cistouretrograma se reserva en pacientes con síntomas durante el día o, bien, con antecedente de infecciones del tracto urinario no evaluados previamente y en quienes padecen alteraciones anatómicas urológicas. El ultrasonido vesical es útil para determinar el volumen de orina residual y el engrosamiento de la pared vesical., ${ }^{3,10,22}$ La radiografía simple de abdomen puede identificar retención fecal, con datos clínicos de estreñimiento, puesto que el tratamiento se asocia con la curación del cuadro de enuresis. ${ }^{23}$ Deben solicitarse estudios neurológicos ante la sospecha de anormalidades 


\begin{tabular}{|c|c|}
\hline Datos clínicos & Posible causa \\
\hline Síntomas durante el día & Disfunción del vaciamiento \\
\hline $\begin{array}{l}\text { Síntomas del tracto urinario inferior (más de } 8 \text { micciones o } \\
\text { menos de } 3 \text { veces por día; hesitancia [retardo miccional], de- } \\
\text { bilidad del chorro urinario, tenesmo, vaciamiento incompleto, } \\
\text { dolor genital o del tracto inferior, goteo posmicción) }\end{array}$ & $\begin{array}{l}\text { Disfunción del vaciamiento o anormalidades } \\
\text { anatómicas }\end{array}$ \\
\hline Periodos prolongados de sequedad (más de 6 meses) & $\begin{array}{l}\text { Enuresis secundaria, frecuentemente asociada con } \\
\text { comorbilidades psicológicas }\end{array}$ \\
\hline Frecuencia de episodios & Enuresis nocturna suele asociarse con persistencia \\
\hline Cambios en la frecuencia o episodios en el tiempo & La historia natural de curación espontánea \\
\hline Volumen aproximado de enuresis & Valorar capacidad vesical \\
\hline Diario del consumo de líquidos & $\begin{array}{c}\text { Puede sugerir poliuria nocturna (incremento en la } \\
\text { tarde-noche; diabetes mellitus, diabetes insípida, } \\
\text { polidipsia psicógena) }\end{array}$ \\
\hline Diario de evacuaciones & $\begin{array}{l}\text { Estreñimiento: puede contribuir a la disminución de la } \\
\text { capacidad vesical }\end{array}$ \\
\hline Revisión de sistemas & $\begin{array}{c}\text { Identificar alteraciones médicas no diagnosticadas que } \\
\text { contribuyen a la enuresis }\end{array}$ \\
\hline - Ronquidos & - Apnea obstructiva del sueño \\
\hline - Pérdida de peso, fatiga & - Diabetes, enfermedad renal \\
\hline - Alteraciones en la marcha & - Disrafismo espinal \\
\hline - Episodios catatónicos & - Desorden de convulsiones \\
\hline - Prurito perianal, vulvovaginitis & - Enfermedades infecciosas \\
\hline - Sed excesiva & - Diabetes, enfermedades renales, polidipsia psicógena \\
\hline Antecedente familiar de enuresis & Factores genéticos \\
\hline
\end{tabular}

en la exploración de columna lumbosacra, del periné y las extremidades inferiores. ${ }^{24}$

\section{Tratamiento}

Es importante sensibilizarse y ser amigable con el niño, además de explicarle las causas del trastorno, con la finalidad de eliminar la culpa y vergüenza. El tratamiento de la enuresis primaria nocturna consiste en la combinación de intervenciones como: educación y entrenamiento, terapia motivacional, implementación de dispositivos de alarma y prescripción de fármacos. ${ }^{1}$ El objetivo del tratamiento incluye: mantenerse seco por las noches, reducir el número de noches húmedas, disminuir la repercusión de la enuresis en el niño y la familia, y evitar la recurrencia del trastorno. ${ }^{25}$ La edad para implementar el tratamiento varía en cada familia, según lo consideren un problema. Para el niño se convierte en un problema significativo cuando interfiere con la capacidad de socializar con personas. ${ }^{26}$ A continuación se analizan las opciones de tratamiento de la enuresis.

\section{Uroterapia}

Implica el entrenamiento para reconocer la zona cortical de la vejiga y enseñar al niño, conscientemente, sobre el control del tracto urinario inferior. El asesoramiento como guía y asistencia para resolver las dificultades persona- 
les, sociales o psicológicas es parte fundamental del tratamiento, pues se ha observado que la implementación de una terapia exitosa en niños no solo incluye aspectos farmacológicos o quirúrgicos. Se ha valorado la terapia cognitiva conductual mediante diversas técnicas, incluida la automonitorización, con resultados favorables. ${ }^{3}$

1. Biofeedback. Es efectivo en la eliminación de desórdenes como: disfunción del vaciamiento, e incluye diversas técnicas que registran la actividad fisiológica y en tiempo real, con emisión de señales acústicas o visuales para que el niño aprenda a reconocer sus propios reflejos. ${ }^{27}$

2. Alarma. Esta técnica se ha utilizado en el tratamiento de la incontinencia nocturna durante muchos años. El Instituto Nacional de la Excelencia Clínica (NICE) realizó uno de los metanálisis más grandes del uso de alarma en niños con enuresis nocturna verdadera versus pacientes control. Los resultados mostraron un incremento cinco veces mayor de permanecer seco durante la noche con el uso de la alarma, comparados con quienes no recibieron tratamiento, con una probabilidad muy baja de recurrencia a 6 meses $(3.6 \%) .^{1}$ Es el tratamiento más efectivo de enuresis nocturna, con los mejores resultados a largo plazo. ${ }^{3}$ Sin embargo, el uso de la alarma puede ser frustrante, pues solo se activa cuando la orina entra en contacto con el sensor, el niño la escucha y debe levantarse al sanitario; los efectos positivos se obtienen entre 2 y 3 semanas. Este tratamiento no es apropiado en niños con enuresis infrecuente (menos de 1-2 episodios por semana) o, bien, en quienes tienen múltiples episodios nocturnos, que resulta en fatiga del paciente y sus padres. ${ }^{28} \mathrm{El}$ objetivo del tratamiento es alcanzar 14 días consecutivos sin episo- dios de enuresis, de lo contrario tendrá que valorarse mediante intervenciones alternativas o intercalar el tratamiento.

3. Desmopresina. Es un análogo de la vasopresina que incrementa la reabsorción de agua en los túbulos distales del riñón, para activar los canales de acuaporina mediante el receptor V2 y disminuir el flujo urinario. ${ }^{1}$ La prescripción de desmopresina representa la primera línea de tratamiento en niños mayores de 5 años, quienes no han tenido reacción satisfactoria con otro tipo de medicamentos, lo que representa una opción para el paciente y sus padres que desean una mejoría a corto plazo o que se niegan al tratamiento con alarma. ${ }^{3} \mathrm{El} 30 \%$ de los pacientes alcanza periodos secos, incluso $40 \%$ logra disminuir los episodios de enuresis con la prescripción de desmopresina; sin embargo, existe recidiva de $60-70 \%$ después de suspender el tratamiento. ${ }^{29}$ Actualmente, su administración es por vía oral durante la noche (la presentación intranasal no se encuentra indicada para el tratamiento de enuresis nocturna, debido a los riesgos de crisis de hiponatremia); la dosis inicial es de $0.2 \mathrm{mg}$ una hora antes de dormir y si en 14 días no hay reacción satisfactoria, puede incrementarse la dosis a $0.4 \mathrm{mg} .{ }^{10}$ Los efectos adversos son poco frecuentes; sin embargo, puede ocurrir hiponatremia dilucional. Para prevenir este efecto se recomienda limitar el consumo de líquidos a $240 \mathrm{~mL}$ una hora antes y 8 horas después de la administración de desmopresina. ${ }^{30}$ El tratamiento debe suspenderse en caso de desequilibrio de líquidos o electrólitos, fiebre, vómito recurrente o diarrea. ${ }^{31}$ La reacción ocurre entre la primera o segunda semanas de iniciar el tratamiento; se indica durante 3 meses, con una semana de descanso; en ese lapso los padres y el paciente pueden valorar la reacción al 
fármaco y si continúan con el tratamiento. 32,33

\section{Tratamiento combinado}

a. Desmopresina y alarma: esta combinación ha demostrado gran efectividad, particularmente en niños con problemas emocionales o de conducta y en quienes han tenido más de un episodio de recurrencia, después del tratamiento con alarma. ${ }^{30}$

b. Desmopresina y oxibutinina: es una combinación efectiva en pacientes con deficiente capacidad vesical y signos de vejiga hiperactiva. Sin embargo, los anticolinérgicos no han demostrado eficacia en el tratamiento de la enuresis verdadera. ${ }^{33-35}$

c. Desmopresina y otros fármacos: el tratamiento en conjunto con indometacina ha demostrado resultados variables. ${ }^{36}$ La combinación de desmopresina y diazepam ha reportado reducción de episodios húmedos. ${ }^{37}$

5. Imipramina. Los antidepresivos tricíclicos disminuyen el periodo REM del sueño, estimulan la secreción de vasopresina y relajan el músculo detrusor. Debido a la eficacia reportada en tratamientos previos, la imipramina representa la tercera línea de tratamiento de la enuresis, principalmente cuando la desmopresina no ha mostrado resultados satisfactorios en combinación con otros fármacos. ${ }^{1}$ Una revisión sistemática reportó que la imipramina disminuyó los episodios a un episodio húmedo por semana y $20 \%$ de los pacientes se mantuvieron secos durante 14 noches consecutivas; sin embargo, el límite de recurrencia fue de $96 \%$ después de suspender el tratamiento. ${ }^{38}$ Imipramina debe administrarse una hora antes de dormir; la presentación comercial es de 10,
25 y $50 \mathrm{mg}$, con dosis inicial de 10-25 mg y puede incrementarse a $25 \mathrm{mg}$ en caso de no obtener reacción satisfactoria después de una semana de iniciar el tratamiento. La dosis estándar en niños de 5-8 años es $25 \mathrm{mg}$ y en niños mayores de $50 \mathrm{mg}$; las dosis no deben exceder los $50 \mathrm{mg}$ en niños de 6-12 años y de $75 \mathrm{mg}$ en niños mayores de 12 años. ${ }^{39} \mathrm{Al}$ igual que el tratamiento con desmopresina, debe valorarse durante 3 meses y de no haber reacción satisfactoria se sugiere suspenderlo gradualmente. Si el tratamiento es exitoso, deberá encontrarse la dosis mínima efectiva. ${ }^{40}$ Los efectos adversos más comunes incluyen alteraciones cardiacas (depresión miocárdica en sobredosis), por lo que algunos pediatras recomiendan efectuar un electrocardiograma antes de indicar el tratamiento e investigar el antecedente familiar de enfermedades cardiacas. ${ }^{1}$

La atención empática y con soporte en la evidencia del médico tratante es parte fundamental del tratamiento de niños con enuresis, y al mismo tiempo los padres deben encontrarse motivados para alcanzar el éxito en la curación de este trastorno.

\section{REFERENCIAS}

1. Anne JW. Childhood enuresis. Paediatr Child Health 2016;26:353-359.

2. Nevéus T, von Gontard A, Hoebeke $P$, et al. The standardization of terminology of lower urinary tract function in children and adolescents: report from the Standardisation Committee of the International Children's Continence Society. J Urol 2006;176(1):314.

3. Israel F, Alexander von G, Mario DG. Evaluation and treatment of nonmonosymptomatic nocturnal enuresis: $A$ standardization document from the International Children's Continence Society. J Pediatr Urol 2013;9:234-243.

4. Fergusson DM, Horwood LJ, Shannon FT. Factors related to the age of attainment of nocturnal bladder control: an 8-year longitudinal study. Pediatrics 1986;78(5):884.

5. Bakker E, van Sprundel M, van der Auwera JC, van Gool JD, Wyndaele JJ. Voiding habits and wetting in a population 
of 4,332 Belgian schoolchildren aged between 10 and 14 years. Scand J Urol Nephrol 2002;36(5):354.

6. Butler RJ, Golding J, Northstone K. Nocturnal enuresis at 7.5 years old: prevalence and analysis of clinical signs. BJU Int 2005;96:404-10.

7. An-Sofie $\mathrm{G}$, Bente S, Olivier O, Piet H. Long-term followup of children with nocturnal enuresis: increased frequency of nocturia in adulthood. J Urol 2014;191:1866-1871.

8. Von Gontard A, Freitag CM, Seifen S, Pukrop R, Röhling D. Neuromotor development in nocturnal enuresis. Dev Med Child Neurol 2006;48(9):744.

9. Watanabe H, Azuma Y. A proposal for a classification system of enuresis based on overnight simultaneous monitoring of electroencephalography and cystometry. Sleep 1989;12(3):257.

10. Robson WL. Clinical practice. Evaluation and management of enuresis. N Engl J Med 2009;360(14):1429.

11. Kawauchi A, Tanaka Y, Naito Y, Yamao Y, Ukimura O, Yoneda K, Mizutani Y, Miki T. Bladder capacity at the time of enuresis. Urology 2003;61(5):1016.

12. Yeung CK, Chiu HN, Sit FK. Bladder dysfunction in children with refractory monosymptomatic primary nocturnal enuresis. J Urol 1999;162(3 Pt 2):1049.

13. Rittig $S$, Schaumburg HL, Siggaard C, Schmidt F, Djurhuus JC. The circadian defect in plasma vasopressin and urine output is related to desmopressin response and enuresis status in children with nocturnal enuresis. J Urol 2008;179(6):2389.

14. L. Dossche, A. Raes, P. Hoebeke, P. Circadian Rhythm of Glomerular Filtration and Solute Handling Related to Nocturnal Enuresis. J Urol 2016;195:162-167.

15. Wolfish NM, Pivik RT, Busby KA. Elevated sleep arousal thresholds in enuretic boys: clinical implications. Acta Paediatr 1997;86(4):381.

16. Hunsballe JM. Increased delta component in computerized sleep electroencephalographic analysis suggests abnormally deep sleep in primary monosymptomatic nocturnal enuresis. Scand J Urol Nephrol 2000;34(5):294.

17. Bader $G$, Nevéus $T$, Kruse $S$, Sillén U. Sleep of primary enuretic children and controls. Sleep 2002;25(5):579.

18. Bakwin $\mathrm{H}$. The genetics of enuresis. In: Bladder control and enuresis. Kolvin RM, Meadows SR, editors. London: Medical Books Ltd, 1973;73.

19. Longstaffe S, Moffatt ME, Whalen JC. Behavioral and selfconcept changes after six months of enuresis treatment: a randomized, controlled trial. Pediatrics 2000;105(4 Pt 2):935.

20. Ullom-Minnich MR. Diagnosis and management of nocturnal enuresis. Am Fam Physician 1996;54(7):2259.

21. Vande Walle J, Rittig S, Bauer S, Eggert P, Marschall-Kehrel D, Tekgul S, American Academy of Pediatrics, European Society for Paediatric Urology, European Society for Paediatric Nephrology, International Children's Continence Society.
Practical consensus guidelines for the management of enuresis. Eur J Pediatr 2012;171(6):971-83.

22. Kovacevic L, Wolfe-Christensen C, Mirkovic J, Yih J, Lakshmanan $Y$. Renal bladder ultrasound evaluation in monosymptomatic primary nocturnal enuresis: is it really necessary?. Pediatr Nephrol 2014;29(7):1189-94.

23. Hodges SJ, Anthony EY. Occult megarectum--a commonly unrecognized cause of enuresis. Urology 2012;79(2):421.

24. Pippi Salle JL, Capolicchio G, Houle AM, Vernet O, Jednak $\mathrm{R}$, O'Gorman AM, Montes JL, Farmer JP. Magnetic resonance imaging in children with voiding dysfunction: is it indicated?. J Urol 1998;160(3 Pt 2):1080.

25. Kiddo D. Nocturnal enuresis. BMJ Clin Evid. 2007;2007. pii:0305.

26. Jalkut MW, Lerman SE, Churchill BM. Enuresis. Pediatr Clin North Am 2001;48(6):1461.

27. Kjolseth D, Knudsen LM, Madsen B, Norgaard JP, Djurhuus JC. Urodynamic biofeedback training for children with bladdersphincter dyscoordination during voiding. Neurourol Urodyn 1993;12:211e21.

28. Kwak KW, Lee YS, Park KH, Baek M. Efficacy of desmopressin and enuresis alarm as first and second line treatment for primary monosymptomatic nocturnal enuresis: prospective randomized crossover study. J Urol 2010;184(6):2521.

29. Wille $\mathrm{S}$. Comparison of desmopressin and enuresis alarm for nocturnal enuresis. Arch Dis Child 1986;61(1):30.

30. Glazener CM, Evans J. Desmopressin for nocturnal enuresis in children. Cochrane Database Syst Rev 2002;(3):CD002112.

31. U.S. Food and Drug Administration, 2007 Safety Alerts for Human Medical Products: Desmopressin Acetate (marketed as DDAVP Nasal Spray, DDAVP Rhinal Tube, DDAVP, DDVP, Minirin, and Stimate Nasal Spray). [En línea]. Dirección URL: <www.fda.gov/Safety/MedWatch/SafetyInformation/SafetyAlertsforHumanMedicalProducts/ ucm152113.htm>. (Consulta: 10 de septiembre 2016).

32. 32. Farida E, Sally Z, Marian K. Assessment of the efficacy of desmopressin in treatment of Primary Monosymptomatic Nocturnal Enuresis in Egyptian children. Egypt J Med Hum Gen. 2015;16:257-262.

33. 33. Farzaneh S, Sepideh S, and Maryam T. Primary monosymptomatic nocturnal enuresis: monotherapy vs combination therapy. Urology 2016;93:170-174.

34. 34. Aaron $B$, Jacqueline $P$, Pamela E. Evaluating use of higher dose oxybutynin in combination with desmopressin for refractory nocturnal enuresis. J Pediatr Urol 2016;12:220. e1-220.e6

35. 35. Austin PF, Ferguson G, Yan Y, Campigotto MJ, Royer $M E$, Coplen DE. Combination therapy with desmopressin and an anticholinergic medication for nonresponders to desmopressin for monosymptomatic nocturnal enuresis: a randomized, double-blind, placebo-controlled trial. Pediatrics 2008;122(5):1027. 
36. Konstantinos K, Søren R, Wendy F. Effect of Indomethacin on Desmopressin Resistant Nocturnal Polyuria and Nocturnal Enuresis. J Urol 2012;188:1915-1923.

37. Deshpande AV, Caldwell PH, Sureshkumar P. Drugs for nocturnal enuresis in children (other than desmopressin and tricyclics). Cochrane Database Syst Rev 2012;12:CD002238.
38. Caldwell PH, Sureshkumar P, Wong WC. Tricyclic and related drugs for nocturnal enuresis in children. Cochrane Database Syst Rev 2016;(1):CD002117.

39. Schmitt BD. Nocturnal enuresis. Pediatr Rev 1997;18(6):183.

40. Gepertz S, Nevéus T. Imipramine for therapy resistant enuresis: a retrospective evaluation. J Urol 2004;171(6 Pt 2):2607.

\section{AVISO PARA LOS AUTORES}

Revista Mexicana de Urología tiene una nueva plataforma de gestión para envío de artículos: https://www.revisionporpares.com/index.php/RMUrol ahí podrá inscribirse a la base de datos administrada por el sistema Open Journal System (OJS) que ofrece las siguientes ventajas para los autores:

- Subir sus artículos directamente al sistema.

- Conocer, en cualquier momento, el estado de los artículos enviados, es decir, si ya fueron asignados a un revisor, aceptados con o sin cambios, o rechazados.

- Participar en el proceso editorial corrigiendo y modificando sus artículos hasta su aceptación final. 